\title{
Supersolid Order from Disorder: Hard-Core Bosons on the Triangular Lattice
}

\author{
R. G. Melko, ${ }^{1}$ A. Paramekanti, ${ }^{2}$ A. A. Burkov, ${ }^{1}$ A. Vishwanath, ${ }^{2}$ D. N. Sheng, ${ }^{3}$ and L. Balents ${ }^{1}$ \\ ${ }^{1}$ Department of Physics, University of California, Santa Barbara, California 93106, USA \\ ${ }^{2}$ Department of Physics, University of California, Berkeley, California 94720, USA \\ ${ }^{3}$ Department of Physics and Astronomy, California State University, Northridge, California 91330, USA
}

(Received 16 May 2005; published 16 September 2005)

\begin{abstract}
We study the interplay of Mott localization, geometric frustration, and superfluidity for hard-core bosons with nearest-neighbor repulsion on the triangular lattice. For this model at half filling, we demonstrate that superfluidity survives for arbitrarily large repulsion, and that diagonal solid order emerges in the strongly correlated regime from an order-by-disorder mechanism. This is thus an unusual example of a stable supersolid phase of hard-core lattice bosons at a commensurate filling.
\end{abstract}

DOI: 10.1103/PhysRevLett.95.127207

Recent experiments on ${ }^{4} \mathrm{He}$ under pressure suggest [1] that a supersolid phase, in which long-range diagonal (crystalline) order and long-range off-diagonal (superfluid) order coexist, may arise at temperatures $T \lesssim 100 \mathrm{mK}$. Such a phase had been theoretically envisioned long ago [2], based on the proposition that a nonvanishing density of zero point defects in the solid (interstitials or vacancies) could Bose condense and form a superfluid "on top" of the existing crystalline background. However, early estimates [3] of the superfluid fraction in clean ${ }^{4} \mathrm{He}$ solid were very small $\left(\sim 10^{-4}\right)$, and recent numerics with realistic interatomic potentials find no evidence for zero point defects or appreciable interparticle exchanges [4] in the crystal.

Since the microscopic conditions under which clean ${ }^{4} \mathrm{He}$ might exist in a supersolid phase are still unclear, it is useful to focus on simple lattice models of bosons in order to understand the different mechanisms by which a supersolid might emerge. Such "lattice supersolids" are superfluids which also break the lattice translation symmetries [5]. Numerical studies of interacting bosons on the square lattice find that stable supersolid phases form upon doping away from a half-filled checkerboard or striped solid [68]. These provide examples of the "defect condensation" mechanism, where doped bosons (holes) act as interstitials (vacancies) in the crystal.

In this Letter we explore a different route to supersolidity based on the competition between Mott localization physics and geometric frustration. To illustrate this, we focus on a simple model of hard-core bosons at half filling on the triangular lattice, interacting via a nearest-neighbor repulsive term,

$$
H=-t \sum_{\langle i j\rangle}\left(b_{i}^{\dagger} b_{j}+b_{j}^{\dagger} b_{i}\right)+\sum_{\langle i j\rangle} V\left(n_{i}-\frac{1}{2}\right)\left(n_{j}-\frac{1}{2}\right) .
$$

We show that a supersolid phase emerges for $V / t \gtrsim 10$, from an order-by-disorder effect in a strongly correlated superfluid. This route to supersolidity is thus complementary to the defect condensation mechanism which starts from a perfect crystal and considers a small defect-density
PACS numbers: 75.10.Jm, 05.30.Jp, 67.40.-w, 67.80.-s

induced supersolid. Our results are based on a combination of analytical arguments, projected boson wave functions, and quantum Monte Carlo and exact diagonalization studies. It would be interesting to look for signatures of this supersolid in experiments, e.g., on interacting cold bosonic atoms in an optical lattice [9], where both a zero momentum condensate peak and Bragg peaks at the ordering wave vectors would be expected in the usual time-of-flight momentum spectroscopy.

Alternatively, the Hamiltonian we study can be viewed, via the standard mapping from hard-core bosons to $S=$ $1 / 2$ spins, as an $X X Z$ model:

$$
H=\sum_{\langle i j\rangle}\left[-J_{\perp}\left(S_{i}^{x} S_{j}^{x}+S_{i}^{y} S_{j}^{y}\right)+J_{z} S_{i}^{z} S_{j}^{z}\right]
$$

with $J_{z}=V$ and $J_{\perp}=2 t$. A spin Hamiltonian such as Eq. (2) may be realized in a Mott insulating phase of cold bosons on an optical lattice if the bosons possess two internal states that play the role of the spin [9]. Since the two-body exchange interaction for spinful bosons is naturally ferromagnetic, $J_{\perp}>0$ in Eq. (2). In spin language, supersolid ordering corresponds to the spins having their $x y$ components aligned ferromagnetically (superfluid) with the $z$ component also ordered at a nonzero wave vector (solid). In our analysis, we will work interchangeably in terms of bosons or spin variables.

Spin wave theory and Landau-Ginzburg-Wilson approach for $J_{\perp} / J_{z} \gg 1$. - For large $J_{\perp} / J_{z}$, the spins align in plane to form an $X Y$ ferromagnetic phase. Ignoring small quantum fluctuations, the ground state has all spins uniformly polarized along the (say) $x$ direction, giving a nonzero $\left\langle S^{x}\right\rangle$. This corresponds to a Bose condensate of the bosons, with off-diagonal long-range order and a nonzero superfluid stiffness. Doing a spin wave expansion about this ordered state, we find that the gap to excitations at wave vectors $\pm \mathbf{k}_{0}= \pm(4 \pi / 3,0)$ (Brillouin zone corners) closes at $J_{z} / J_{\perp}=2$. In the boson language, the "roton minimum" of the superfluid hits zero energy. The uniform superfluid develops an instability toward 3-sublattice or- 
dering, $S_{i}^{z} \sim \operatorname{Re}\left(\psi e^{i \mathbf{k}_{0} \cdot \mathbf{r}_{i}}\right)$, with the order parameter $\psi$ describing the critical modes. The Landau-Ginzburg-Wilson (LGW) expansion of the effective action in $\psi$ and the uniform Ising magnetization $M$ has the form:

$$
\begin{aligned}
S= & \int d^{2} x \int_{0}^{\beta} d \tau\left[\left|\partial_{\tau} \psi\right|^{2}+c^{2}|\nabla \psi|^{2}+r|\psi|^{2}+u|\psi|^{4}\right. \\
& \left.+v|\psi|^{6}+w \operatorname{Re}\left(\psi^{6}\right)+M^{2} /(2 \chi)-\lambda M \operatorname{Re}\left(\psi^{3}\right)\right],
\end{aligned}
$$

where the constant $\chi$ represents the boson compressibility, and $\lambda$ is generally nonzero from symmetry considerations. This LGW action describes the onset of nonzero $\psi$ within the superfluid; i.e., the ordered state is a supersolid. Exhaustive evidence supporting this interpretation will be provided below.

The "massive" $M$ field can be integrated out to obtain an effective action for $\psi$ alone, with the renormalized parameters $\tilde{v}=v-\chi \lambda^{2} / 2, \tilde{w}=w-\chi \lambda^{2} / 2$. Two possible ordered states thereby occur, determined by the sign of $\tilde{w}$. For $\tilde{w}<0, \psi^{3}$ is purely real, and from Eq. (3), a nonzero spontaneous uniform magnetization $M$ is predicted. This ferrimagnetic state has a three-sublattice structure with $\left\langle S_{i}^{z}\right\rangle=\left(m, m,-m^{\prime}\right) \quad\left(M=\left(2 m-m^{\prime}\right) / 3 \neq 0\right)$, and requires phase separation in the canonical boson number ensemble. For $\tilde{w}>0, \psi^{3}$ is purely imaginary [10], and $M=0$. The corresponding three-sublattice pattern has $\left\langle S_{i}^{z}\right\rangle=(m,-m, 0)$, so we refer to this as an antiferromagnetic state (no phase separation is implied). The instability to three-sublattice ordering was also found by Murthy et al. [11] who suggested a supersolid phase within spin wave theory based on a large- $S$ expansion. Here we provide a more thorough and rigorous analysis, directly for the $S=$ $1 / 2$ case of most interest. Note also that Eq. (3) is an appropriate starting point for investigating the quantum critical behavior at the zero-temperature superfluidsupersolid transition.

Variational wave function. - A simple variational wave function argument indicates that superfluidity survives in our model for arbitrarily small $J_{\perp} / J_{z}$. A good variational approximation to the ground state for large $J_{\perp} / J_{z}$ is the hard-core projection of the noninteracting Bosecondensate wave function, $\Psi_{0}\left(\mathbf{r}_{1}, \ldots, \mathbf{r}_{N_{b}}\right)=1$ if all $\mathbf{r}_{i}$ are distinct and zero otherwise, where $N_{b}$ is half of the number of sites $N$. To account for intersite correlations with increasing $J_{z}$, we incorporate a Jastrow factor which leads to

$$
\Psi(\{\mathbf{r}\}, g)=\left[\prod_{\langle i, j\rangle}(1-g)^{\left(n_{i}-1 / 2\right)\left(n_{j}-1 / 2\right)}\right] \Psi_{0}(\{\mathbf{r}\}),
$$

where $0 \leq g<1$ is a variational parameter, and $\{\mathbf{r}\} \equiv$ $\left(\mathbf{r}_{1} \ldots \mathbf{r}_{N_{b}}\right)$. Using a Monte Carlo method, we have evaluated correlation functions in $\Psi$ with the optimal $g \equiv g^{*}$ at each value of $J_{z} / J_{\perp}$.

For $J_{z}=0$, we find $g^{*} \approx 0.2$, and the wave function in Eq. (4) has off-diagonal long-range order, with $\left\langle b_{i}\right\rangle \approx$ 0.48 . Imposing a phase gradient $\phi$ in the wave function
$\Psi$ through a twist in the boundary condition, the superfluid stiffness is defined as

$$
\rho_{s}=\frac{\partial^{2} E}{\partial \phi^{2}}=\lim _{\phi \rightarrow 0} \frac{\Delta E}{N} \frac{2}{\phi^{2}},
$$

where $\Delta E$ is the resulting change in energy. Using this definition, we estimate $\rho_{s}\left(J_{z}=0\right) \approx 0.54 J_{\perp}$. With increasing $J_{z}$, we find $g^{*}$ increases and the superfluid density decreases, as illustrated in Fig. 1(a).

Let us go to the most interesting limit of $J_{\perp} / J_{z} \rightarrow 0$. For $J_{\perp}=0$, the $X X Z$ model in Eq. (2) reduces to the classical Ising antiferromagnet on a triangular lattice which is well known to have a macroscopic degeneracy of ground states $\approx 1.381^{N}$. These are configurations with exactly one frustrated bond per triangle [12]. For $J_{z} \rightarrow \infty$, we find the optimal $g^{*} \rightarrow 1$, and the wave function $\Psi(\{\mathbf{r}\}, 0)$ is then an equal weight linear superposition of all Ising ground state configurations with $S_{\text {tot }}^{z}=0$. In this case $\left\langle b_{i}\right\rangle \approx 0.13$, and the superfluid stiffness $\rho_{s} \approx 0.09 J_{\perp}$ [see Fig. 1(a)]. This persistence of off-diagonal long-range order on the triangular lattice for $J_{\perp} / J_{z} \rightarrow 0$ is one of the most important points of this Letter.

Remarkably, despite its simplicity, the variational wave function with $g \rightarrow 1$ can also be shown (analytically and numerically) to have power-law $1 / \sqrt{r}$ correlations of $S_{i}^{z}$ at the wave vectors $\pm \mathbf{k}_{0}$, in addition to off-diagonal order, reminiscent of the long-range Ising correlations expected from the LGW theory. A variational wave function for the supersolid phase with true long-range density order is currently being investigated.

Strong-coupling analysis for $J_{\perp} / J_{z} \ll 1$. -We now turn to directly consider the strong-coupling limit. A simple

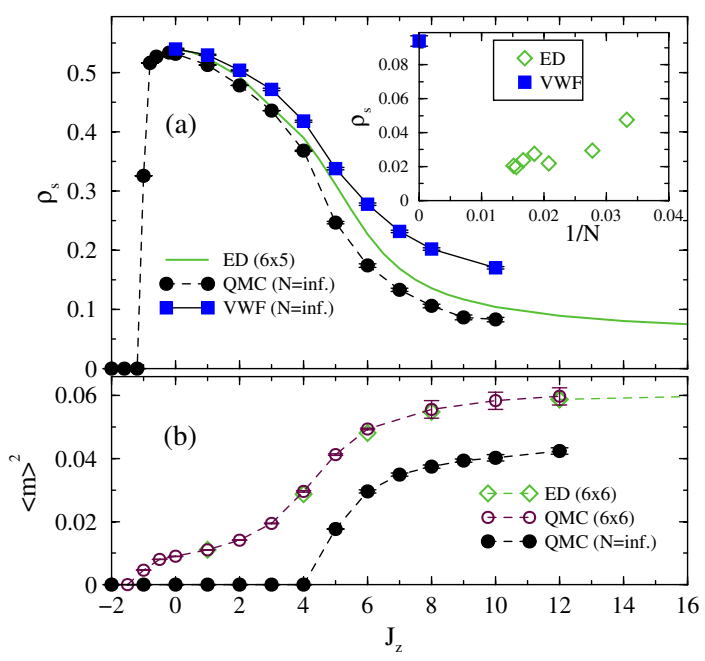

FIG. 1 (color online). (a) Ground state superfluid density, calculated with exact diagonalization, quantum Monte Carlo, and variational wave function, with $J_{\perp}=1$. The inset is $\rho_{s}$ in the limit $J_{z} \rightarrow \infty$, calculated by ED (for several system sizes $N$ ) and VWF (in the thermodynamic limit). (b) The diagonal order parameter squared. Examples of the QMC extrapolations to $N \rightarrow \infty$ are illustrated in Fig. 2 . 
qualitative picture (which we will reinforce with a rigorous analysis a little later) can be obtained by mapping the $X X Z$ model onto a transverse field Ising model:

$$
H=J_{z} \sum_{\langle i j\rangle} S_{i}^{z} S_{j}^{z}-h_{\mathrm{eff}} \sum_{i} S_{i}^{x},
$$

with $h_{\text {eff }}=J_{\perp}\left\langle S_{i}^{x}\right\rangle$. We have assumed here that, as suggested by the variational wave function analysis, superfluidity survives in the limit of small $J_{\perp} / J_{z}$. This transverse field Ising model has been studied earlier $[10,13]$ in various contexts, using Ginzburg-Landau theory and Monte Carlo numerics. It is well established that this model has diagonal long-range order, in particular, of antiferromagnetic type, for small $h_{\text {eff }} / J_{z}$. This analogy suggests that the supersolid behavior of the $X X Z$ model should be viewed, similarly to Eq. (6), as arising microscopically from an order-bydisorder mechanism [14].

One might worry that the above analysis relied on a mean-field treatment of the superfluid component of the order, which may be unreliable at small $J_{\perp} / J_{z}$. We now turn to an exact argument that strongly supports a ground state with broken spatial symmetries. In particular, we prove that for $J_{\perp} / J_{z} \rightarrow 0$, the ground states are threefold degenerate and transform nontrivially under lattice rotations.

In this limit $\left(J_{z}=\infty\right)$, the $X X Z$ model reduces to

$$
H_{\infty}=-J_{\perp} \sum_{\langle i j\rangle} \hat{P}_{C}\left(S_{i}^{x} S_{j}^{x}+S_{i}^{y} S_{j}^{y}\right) \hat{P}_{C},
$$

where $\hat{P}_{C}$ is a projection operator onto the classical Ising antiferromagnetic ground state manifold. Consider an $L \times L$ system with periodic boundary conditions, i.e., on the torus. We define the quantities $E_{a} \geq 0, a=1,2,3$, as the number of frustrated bonds along a straight closed path (loop) through $L$ sites along the $a$ th principal axis of the triangular lattice. These quantities are independent of translations of the loop. Moreover, it is straightforward to show that they commute with $H_{\infty}$ in Eq. (7) (and indeed any local Hamiltonian in this manifold). Because each triangle has one frustrated bond, one also finds $E_{1}+E_{2}+E_{3}=L$. Under a sixfold rotation of the system, the $E_{a}$ cyclically permute. Hence a unique ground state with $E_{1}=E_{2}=E_{3}$ that is also rotationally invariant can be found only for $L$ a multiple of three. For $L \neq 0(\bmod 3)$, the ground state thus forms at least a threefold degenerate rotational multiplet, as promised [it can be spanned by three states with "angular momentum" $\ell=0,1,2(\bmod 3)]$.

More refined arguments indicate that this degeneracy is split only by terms of $O\left(e^{-c L}\right.$ ) (with $c$ independent of $L$ ) for $0<J_{\perp} / J_{z} \ll 1$, and $L \rightarrow \infty$. Hence a nontrivial rotational multiplet remains the ground state with exponential accuracy in the thermodynamic limit. This behavior is inconsistent with a simple uniform superfluid, which is expected to have only states with excitation energies above the ground state scaling as a power law of $L$. The most natural possibility consistent with this behavior is broken spatial symmetry of the ground state in the thermodynamic limit. Having concluded that the system must break lattice symmetries, and already argued from our variational wave function that superfluidity persists for $J_{z} \rightarrow \infty$, we thus come to the conclusion that the ground state at large $J_{z}$ is a supersolid, as our numerical simulations below prove.

Exact numerical results. - To address the properties of the spin-1/2 $X X Z$ model in Eq. (2) we have carried out exact diagonalization (ED) studies, as well as stochastic series expansion (SSE) quantum Monte Carlo (QMC) simulations [16], in the grand canonical framework. Using straightforward modifications [15] to the original SSE directed-loop algorithm [17], the QMC is able to explore all regions of the finite-temperature phase diagram for small $J_{z} / J_{\perp}$. However, for $J_{z} / J_{\perp} \geqslant 10$, the QMC is observed to experience a dynamical freezing due to the development of large energy barriers for local updates. In Fig. 1, we summarize our results for ED over the entire range of coupling strengths, and QMC in the weak to intermediate coupling regime (where we have been able to reliably equilibriate data).

The superfluid density is estimated by the stiffness, Eq. (5), easily measured using winding numbers in the QMC simulations $[17,18]$. For a given $J_{z} / J_{\perp}, \rho_{s}$ is extrapolated to $L \rightarrow \infty$ using finite-size scaling on systems of size $N=L \times L$ [Fig. 2(a)]. The results show that for small $J_{z} / J_{\perp}$ the system is clearly in a robust superfluid ground state with a large $\rho_{s}$. The stiffness value decreases with increasing $J_{z} / J_{\perp}$; however, it remains finite to the largest values of $J_{z} / J_{\perp}$ studied with the QMC [19]. The survival

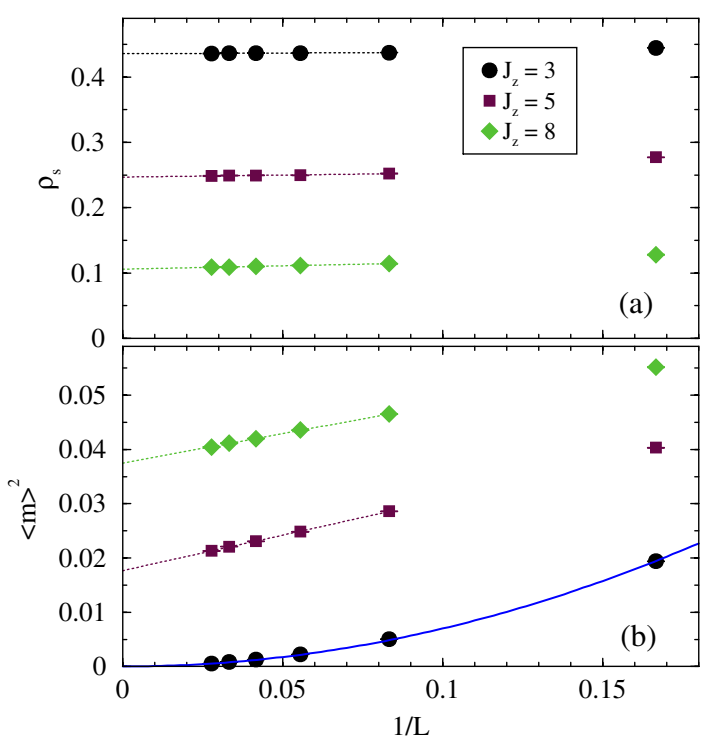

FIG. 2 (color online). Finite-size scaling of QMC data for $\rho_{s}$ (a) and $\langle m\rangle^{2}=S\left(\mathbf{k}_{0}\right) / L^{2}$ (b). Here, $J_{\perp}=1$ and the inverse temperature is $\beta=10$. Error bars (illustrated) are much smaller than the symbol sizes. Dotted lines show linear extrapolations for the five largest system sizes. The thick solid (blue) line is a fit to $\langle m\rangle^{2} \propto 1 / L^{2}$. 
of $\rho_{s}$ at large $J_{z} / J_{\perp}$ is in stark contrast to the behavior at $J_{z} / J_{\perp}=-1$, where the phase transition to a fully polarized ferromagnetic phase is evident by the vanishing stiffness. Further, ED calculations [Fig. 1(a), inset] strongly suggest that $\rho_{s}$ survives to $J_{z} / J_{\perp} \rightarrow \infty$, although system sizes are too small to allow a reliable extrapolation to $N \rightarrow \infty$ for these data. The small value of $\rho_{s}$ for $J_{z} \rightarrow \infty$ indicates that the supersolid is very close in parameter space to an insulating state.

In the above analytical arguments, the survival of $\rho_{s}$ at large $J_{z} / J_{\perp}$ is accompanied by the development of simultaneous Ising order (the supersolid phase). To address this prediction, we compute the static structure factor

$$
S(\mathbf{k})=\frac{1}{N} \sum_{\langle i j\rangle} e^{i \mathbf{k} \cdot\left(\mathbf{r}_{i}-\mathbf{r}_{j}\right)}\left\langle S_{i}^{z} S_{j}^{z}\right\rangle,
$$

which is found to develop sharp Bragg peaks for moderate values of $J_{z} / J_{\perp}$. The peaks occur at the corners of the hexagonal Brillouin zone [ $\left.\pm \mathbf{k}_{0}= \pm(4 \pi / 3,0)\right]$, in complete agreement with the above analytical discussion. The presence of diagonal long-range order (LRO) can be demonstrated rigorously through a finite-size scaling analysis of $S\left(\mathbf{k}_{0}\right)$ [Fig. 2(b)]. Specifically, in a LRO state, the diagonal order parameter squared, $\langle m\rangle^{2}=S\left(\mathbf{k}_{0}\right) / L^{2}$, should scale to a constant as $L \rightarrow \infty$ [with a leading-order correction varying as $1 / L[20]]$. In contrast, in the absence of diagonal LRO, spin-spin correlations are short ranged, and $\langle m\rangle^{2}$ should tend to zero as $1 / L^{2}$ in the asymptotic limit. Employing this analysis on the QMC data for all $J_{z} / J_{\perp}$ studied reveals the position of the zero-temperature superfluid-supersolid phase transition to be between $J_{z} / J_{\perp}=4$ and $J_{z} / J_{\perp}=5$ (Fig. 1).

Finally, we note that the grand canonical QMC simulations indicate no significant deviations from half filling in the supersolid phase for system sizes up to $L=36$. In the $\mathrm{ED}$, the minimum-energy ground state is also found to lie within the $\left\langle S^{z}\right\rangle=0$ subspace. These observations suggest the occurrence of the antiferromagnetic rather than the ferrimagnetic supersolid; however, QMC simulations on much larger system sizes may be necessary in order to distinguish conclusively between the two.

Conclusions. - We have presented a comprehensive study of the phase diagram of interacting hard-core bosons at half filling (zero field $X X Z$ model) on the triangular lattice. We have uncovered a remarkable order-by-disorder route to a supersolid phase in this model. This mechanism relies on a subtle interplay between strong repulsion and quantum fluctuations. In some square lattice models, defect condensation results in a supersolid that is present away from half filling, but unstable to phase separation otherwise [8]. In contrast, the order-by-disorder mechanism permits a stable supersolid even at (or very close to) half filling, since this density maximizes the entropy of the frustrated manifold of $S^{z}$ configurations.

The authors would like to thank A.W. Sandvik, D. J. Scalapino, and D. Stamper-Kurn for insightful dis- cussions. Supercomputer time was provided by NCSA under Grant No. DMR020029N. Financial support was provided by National Science Foundation Grants No. DMR02-11166 (R. G. M.), No. DMR-9985255 (L. B., A. A. B.), No. DMR-0307170 (D.N.S.), the Packard Foundation (L.B., A. A.B.), ACS-PRF 41752-AC10 (D. N. S.), and DOE LDRD DE-AC03-76SF00098 (A.P. and A. V.). We would like to thank the authors of Ref. [21], especially K. Damle, for correspondence comparing and contrasting our results.

Note added.-Recently we became aware of parallel numerical works [21] that reach similar conclusions.

[1] E. Kim and M. H. W. Chan, Nature (London) 427, 225 (2004); Science 305, 1941 (2004).

[2] A. F. Andreev and I. M. Lifshitz, Sov. Phys. JETP 29, 1107 (1969); G. Chester, Phys. Rev. A 2, 256 (1970).

[3] A. J. Leggett, Phys. Rev. Lett. 25, 1543 (1970).

[4] D. M. Ceperley and B. Bernu, Phys. Rev. Lett. 93, 155303 (2004).

[5] K.-S. Liu and M.E. Fisher, J. Low Temp. Phys. 10, 655 (1973).

[6] A. van Otterlo and K.-H. Wagenblast, Phys. Rev. Lett. 72, 3598 (1994); A. van Otterlo et al., Phys. Rev. B 52, 16176 (1995).

[7] G. G. Batrouni and R. T. Scalettar, Phys. Rev. Lett. 84, 1599 (2000); F. Hebert et al., Phys. Rev. B 65, 014513 (2002).

[8] P. Sengupta, L. P. Pryadko, F. Alet, M. Troyer, and G. Schmidt, Phys. Rev. Lett. 94, 207202 (2005).

[9] L.-M. Duan, E. Demler, and M. D. Lukin, Phys. Rev. Lett. 91, 090402 (2003).

[10] D. Blankschtein, M. Ma, A. N. Berker, G. S. Grest, and C. M. Soukoulis, Phys. Rev. B 29, R5250 (1984).

[11] G. Murthy, D. Arovas, and A. Auerbach, Phys. Rev. B 55, 3104 (1997).

[12] G. H. Wannier, Phys. Rev. 79, 357 (1950); R. M.F. Houtappel, Physica (Amsterdam) 16, 425 (1950).

[13] R. Moessner and S. L. Sondhi, Phys. Rev. B 63, 224401 (2001); R. Moessner, S. L. Sondhi, and P. Chandra, Phys. Rev. B 64, 144416 (2001).

[14] In contrast, generalizations of our analytical arguments predict a simple uniform superfluid on the kagome lattice at all $J_{z} / J_{\perp}$. This prediction has recently been confirmed by QMC calculations [15].

[15] S. V. Isakov and R. G. Melko (to be published).

[16] A. W. Sandvik and J. Kurkijärvi, Phys. Rev. B 43, 5950 (1991); A. W. Sandvik, J. Phys. A 25, 3667 (1992).

[17] O.F. Syljuåsen and A. W. Sandvik, Phys. Rev. E 66, 046701 (2002).

[18] E. L. Pollock and D. M. Ceperley, Phys. Rev. B 36, 8343 (1987).

[19] M. Boninsegni, J. Low Temp. Phys. 132, 39 (2003).

[20] D. A. Huse, Phys. Rev. B 37, R2380 (1988).

[21] D. Heidarian and K. Damle, this issue, Phys. Rev. Lett. 95, 127206 (2005); S. Wessel and M. Troyer, this issue, Phys. Rev. Lett. 95, 127205 (2005). 